\title{
Bayesian Prediction of Future Generalized Order Statistics from a Class of Finite Mixture Distributions
}

\author{
Abd EL-Baset A. Ahmad', Areej M. Al-Zaydi'2 \\ ${ }^{1}$ Department of Mathematics, Assiut University, Assiut, Egypt \\ ${ }^{2}$ Department of Mathematics, Taif University, Taif, Saudi Arabia \\ Email: abahmad2002@yahoo.com, aree.m.z@hotmail.com
}

Received 9 August 2015; accepted 25 October 2015; published 28 October 2015

Copyright (C) 2015 by authors and Scientific Research Publishing Inc.

This work is licensed under the Creative Commons Attribution International License (CC BY). http://creativecommons.org/licenses/by/4.0/

(c) (i) Open Access

\section{Abstract}

This article is concerned with the problem of prediction for the future generalized order statistics from a mixture of two general components based on doubly type II censored sample. We consider the one sample prediction and two sample prediction techniques. Bayesian prediction intervals for the median of future sample of generalized order statistics having odd and even sizes are obtained. Our results are specialized to ordinary order statistics and ordinary upper record values. A mixture of two Gompertz components model is given as an application. Numerical computations are given to illustrate the procedures.

\section{Keywords}

Generalized Order Statistics, Bayesian Prediction, Heterogeneous Population, Doubly Type II Censored Samples, One- and Two-Sample Schemes

\section{Introduction}

Let the random variable (rv) $T$ follows a class including some known lifetime models; its cumulative distribution function (CDF) is given by

$$
F(t)=1-\exp [-\theta \lambda(t)], t>0,(\theta>0),
$$

and its probability density function (PDF) is given by

$$
f(t)=\theta \lambda^{\prime}(t) \exp [-\theta \lambda(t)], t>0,(\theta>0),
$$


where $\lambda^{\prime}(t)$ is the derivative of $\lambda(t)$ with respect to $t$ and $\lambda(t) \equiv \lambda(t ; \alpha)$ is a nonnegative continuous function of $t$ and $\alpha$ may be a vector of parameters, such that

$$
\lambda(t) \rightarrow 0 \text { as } t \rightarrow 0^{+} \text {and } \lambda(t) \rightarrow \infty \text { as } t \rightarrow \infty \text {. }
$$

The reliability function (RF) and hazard rate function (HRF) are given, respectively, by

$$
\begin{gathered}
R(t)=\exp [-\theta \lambda(t)], \\
H(t)=\theta \lambda^{\prime}(t),
\end{gathered}
$$

where $H()=.f(.) / R($.$) .$

The general problem of statistical prediction may be described as that of inferring the value of unknown observable that belongs to a future sample from current available information, known as the informative sample. As in estimation, a predictor can be either a point or an interval predictor. The problem of prediction can be solved fully within Bayesian framework [1].

Prediction has been applied in medicine, engineering, business and other areas as well. For details on the history of statistical prediction, analysis, application and examples see for example [1] [2].

Bayesian prediction of future order statistics and records from different populations has been dealt with by many authors. Among others, [3] predicted observables from a general class of distributions. [4] obtained Bayesian prediction bounds under a mixture of two exponential components model based on type I censoring. [5] obtained Bayesian predictive survival function of the median of a set of future observations. Bayesian prediction bounds based on type I censoring from a finite mixture of Lomax components were obtained by [6]. [7] obtained Bayesian predictive density of order statistics based on finite mixture models. [8] obtained Bayesian interval prediction of future records. Based on type I censored samples, Bayesian prediction bounds for the $s^{\text {th }}$ future observable from a finite mixture of two component Gompertz life time model were obtained by [9]. [10] considered Bayes inference under a finite mixture of two compound Gompertz components model. Bayesian prediction of future median has been studied by, among others, they were [5] [11] [12].

Recently, [13] introduced the generalized order statistics (GOS'S). Ordinary order statistics, ordinary record values and sequential order statistics were, among others, special cases of GOS'S. For various distributional properties of GOS'S, see [13]. The GOS'S have been considered extensively by many authors, among others, they were [14]-[33].

Mixtures of distributions arise frequently in life testing, reliability, biological and physical sciences. Some of the most important references that discuss different types of mixtures of distributions are a monograph by [34]-[36].

The PDF, CDF, RF and HRF of a finite mixture of two components of the class under study are given, respectively, by

$$
\begin{gathered}
f(t)=p_{1} f_{1}(t)+p_{2} f_{2}(t), \\
F(t)=p_{1} F_{1}(t)+p_{2} F_{2}(t), \\
R(t)=p_{1} R_{1}(t)+p_{2} R_{2}(t), \\
H(t)=f(t) / R(t),
\end{gathered}
$$

where, for $j=1,2$, the mixing proportions $p_{j}$ are such that $0 \leq p_{j} \leq 1, p_{1}+p_{2}=1$ and $f_{j}(t), F_{j}(t), R_{j}(t)$ are given from (1), (2), (3) after using $\theta_{j}$ and $\lambda_{j}(t)$ instead of $\theta$ and $\lambda(t)$.

The property of identifiability is an important consideration on estimating the parameters in a mixture of distributions. Also, testing hypothesis, classification of random variables, can be meaning fully discussed only if the class of all finite mixtures is identifiable. Idenifiability of mixtures has been discussed by several authors, including [37]-[39].

This article is concerned with the problem of obtaining Bayesian prediction intervals (BPI) for the future GOS'S from a mixture of two general components based on doubly type II censored sample. One- and two-sample prediction cases are treated in Sections 2 and 3, respectively. Bayesian prediction intervals for the median of future sample of GOS'S having odd and even sizes are obtained in Sections 4. A mixture of two Gompertz components is given as an application in Section 5. Finally, numerical computations are given in Section 6. 


\section{One Sample Prediction}

Let $T_{s ; n, m, k}, T_{s+1 ; n, m, k}, \cdots, T_{r ; n, m, k}, 1 \leq s<r \leq n, k>0$ be the $(r-s)$ GOS'S drawn from a mixture of two components of the class (2). Based on this doubly censored sample, the likelihood function can be written (see [27]) as

$$
L(\theta \mid \boldsymbol{t})=\left\{\begin{array}{l}
c_{1}\left\{\prod_{i=s}^{r}\left[R\left(t_{i}\right)\right]^{m} f\left(t_{i}\right)\right\}\left[R\left(t_{r}\right)\right]^{\gamma_{r+1}} \times \sum_{\ell=0}^{s-1} \omega_{\ell}^{(s)}\left[R\left(t_{s}\right)\right]^{(s-\ell-1)(m+1)}, \quad m \neq-1, \\
c_{2}\left[R\left(t_{r}\right)\right]^{k}\left[\ln R\left(t_{s}\right)\right]^{s-1} \prod_{i=s}^{r} H\left(t_{i}\right), \quad m=-1,
\end{array}\right.
$$

where $\boldsymbol{t}=\left(t_{s}, \cdots, t_{r}\right), \quad \theta \in \Theta, \Theta$ is the parameter space, and

$$
\left.\begin{array}{l}
c_{1}=\frac{(-1)^{s-1} C_{r-1}}{(m+1)^{s-1}(s-1) !}, c_{2}=\frac{(-1)^{s-1} k^{r}}{(s-1) !}, \\
C_{r-1}=\prod_{j=1}^{r} \gamma_{j}, \gamma_{r}=k+(n-r)(m+1), \\
\omega_{\ell}^{(s)}=(-1)^{\ell}\left(\begin{array}{c}
s-1 \\
\ell
\end{array}\right) .
\end{array}\right\}
$$

For definition and various distributional properties of GOS'S, see [13].

By substituting Equations (1) and (5) in Equation (9), we get

for $m \neq-1$,

$$
\begin{aligned}
L(\theta \mid \boldsymbol{t})= & c_{1}\left\{\prod_{i=s}^{r}\left[p_{1} R_{1}\left(t_{i}\right)+p_{2} R_{2}\left(t_{i}\right)\right]^{m}\left[p_{1} f_{1}\left(t_{i}\right)+p_{2} f_{2}\left(t_{i}\right)\right]\right\} \\
& \times\left[p_{1} R_{1}\left(t_{r}\right)+p_{2} R_{2}\left(t_{r}\right)\right]^{\gamma_{r+1}} \sum_{\ell=0}^{s-1} \omega_{\ell}^{(s)}\left[p_{1} R_{1}\left(t_{s}\right)+p_{2} R_{2}\left(t_{s}\right)\right]^{(s-\ell-1)(m+1)} .
\end{aligned}
$$

And for $m=-1$,

$$
L(\theta \mid \boldsymbol{t})=c_{2}\left[p_{1} R_{1}\left(t_{r}\right)+p_{2} R_{2}\left(t_{r}\right)\right]^{k}\left(\ln \left[p_{1} R_{1}\left(t_{s}\right)+p_{2} R_{2}\left(t_{s}\right)\right]\right)^{s-1} \times \prod_{i=s}^{r} \frac{\left[p_{1} f_{1}\left(t_{i}\right)+p_{2} f_{2}\left(t_{i}\right)\right]}{\left[p_{1} R_{1}\left(t_{i}\right)+p_{2} R_{2}\left(t_{i}\right)\right]} .
$$

We shall use the conjugate prior density, that was suggested by [3], in the following form

$$
\pi(\theta ; v) \propto C(\theta ; v) \exp [-D(\theta ; v)], \theta=\left(p, \theta_{1}, \theta_{2}, \alpha_{1}, \alpha_{2}\right), v \in \Omega,
$$

where $\Omega$ is the hyper parameter space.

Then the posterior PDF of $\theta, \pi^{*}(\theta \mid \boldsymbol{t})$, is given by

$$
\pi^{*}(\theta \mid \boldsymbol{t}) \propto \pi(\theta ; v) L(\theta \mid \boldsymbol{t}) .
$$

Substituting from Equations (10) and (12) in Equation (13), for $m \neq-1$, the posterior PDF $\pi^{*}(\theta \mid \boldsymbol{t})$ takes the form

$$
\begin{aligned}
\pi^{*}(\theta \mid \boldsymbol{t}) \propto & C(\theta ; v) \exp [-D(\theta ; v)]\left\{\prod_{i=s}^{r}\left[p_{1} R_{1}\left(t_{i}\right)+p_{2} R_{2}\left(t_{i}\right)\right]^{m}\left[p_{1} f_{1}\left(t_{i}\right)+p_{2} f_{2}\left(t_{i}\right)\right]\right\} \\
\times & {\left[p_{1} R_{1}\left(t_{r}\right)+p_{2} R_{2}\left(t_{r}\right)\right]^{\gamma_{r+1}} \sum_{\ell=0}^{s-1} \omega_{\ell}^{(s)}\left[p_{1} R_{1}\left(t_{s}\right)+p_{2} R_{2}\left(t_{s}\right)\right]^{\delta_{\ell}^{(s)}} }
\end{aligned}
$$

where $\delta_{\ell}^{(s)}=(s-\ell-1)(m+1)$.

For $m=-1$, using Equations (11) and (12) in Equation (13), the posterior PDF can be written as 


$$
\begin{aligned}
& \pi^{*}(\theta \mid \boldsymbol{t}) \propto C(\theta ; v) \exp [-D(\theta ; v)]\left[p_{1} R_{1}\left(t_{r}\right)+p_{2} R_{2}\left(t_{r}\right)\right]^{k} \\
& \times\left(\ln \left[p_{1} R_{1}\left(t_{s}\right)+p_{2} R_{2}\left(t_{s}\right)\right]\right)^{s-1} \prod_{i=s}^{r}\left[p_{1} f_{1}\left(t_{i}\right)+p_{2} f_{2}\left(t_{i}\right)\right] \\
& {\left[p_{1} R_{1}\left(t_{i}\right)+p_{2} R_{2}\left(t_{i}\right)\right] }
\end{aligned} .
$$

Now, suppose that the first $(r-s)$ GOS'S $T_{s ; n, m, k}, T_{s+1 ; n, m, k}, \cdots, T_{r ; n, m, k}, 1 \leq s<r \leq n$, have been formed and we wish to predict the future GOS'S $T_{r+1 ; n, m, k}, T_{r+2 ; n, m, k}, \cdots, T_{n ; n, m, k}$. Let $T_{a}^{*} \equiv T_{r+a ; n, m, k}, \quad a=1,2, \cdots, n-r$, the conditional PDF of the $a^{\text {th }}$ future GOS given the past observations $\boldsymbol{t}$, can be written (see [27]) as

$$
h\left(t_{a}^{*} \mid \theta, \boldsymbol{t}\right) \propto\left\{\begin{array}{l}
\sum_{i=0}^{a-1} \omega_{i}^{(a)}\left[R\left(t_{a}^{*}\right)\right]^{\gamma_{r+a-i}-1}\left[R\left(t_{r}\right)\right]^{-\gamma_{r+a-i}} f\left(t_{a}^{*}\right), m \neq-1, \\
\sum_{i=0}^{a-1} \omega_{i}^{(a)}\left[\ln R\left(t_{a}^{*}\right)\right]^{i}\left[\ln R\left(t_{r}\right)\right]^{a-i-1}\left[R\left(t_{a}^{*}\right)\right]^{k-1}\left[R\left(t_{r}\right)\right]^{-k} f\left(t_{a}^{*}\right), m=-1,
\end{array}\right.
$$

where $\omega_{i}^{(a)}=(-1)^{i}\left(\begin{array}{c}a-1 \\ i\end{array}\right)$.

When $m \neq-1$, substituting from Equations (1) and (5) in Equation (16), the conditional PDF takes the form

$$
\begin{aligned}
h\left(t_{a}^{*} \mid \theta, \boldsymbol{t}\right) \propto & \sum_{i=0}^{a-1} \omega_{i}^{(a)}\left[p_{1} R_{1}\left(t_{a}^{*}\right)+p_{2} R_{2}\left(t_{a}^{*}\right)\right]^{\gamma_{r+a-i}-1} \\
& \times\left[p_{1} R_{1}\left(t_{r}\right)+p_{2} R_{2}\left(t_{r}\right)\right]^{-\gamma_{r+a-i}}\left[p_{1} f_{1}\left(t_{a}^{*}\right)+p_{2} f_{2}\left(t_{a}^{*}\right)\right],
\end{aligned}
$$

In the case when $m=-1$; the conditional PDF takes the form

$$
\begin{aligned}
h\left(t_{a}^{*} \mid \theta, \boldsymbol{t}\right) \propto & \sum_{i=0}^{a-1}\left\{\omega_{i}^{(a)}\left(\ln \left[p_{1} R_{1}\left(t_{a}^{*}\right)+p_{2} R_{2}\left(t_{a}^{*}\right)\right]\right)^{i}\left(\ln \left[p_{1} R_{1}\left(t_{r}\right)+p_{2} R_{2}\left(t_{r}\right)\right]\right)^{a-i-1}\right\} \\
& \times\left[p_{1} R_{1}\left(t_{a}^{*}\right)+p_{2} R_{2}\left(t_{a}^{*}\right)\right]^{k-1}\left[p_{1} R_{1}\left(t_{r}\right)+p_{2} R_{2}\left(t_{r}\right)\right]^{-k}\left[p_{1} f_{1}\left(t_{a}^{*}\right)+p_{2} f_{2}\left(t_{a}^{*}\right)\right],
\end{aligned}
$$

The predictive PDF of $T_{a}^{*}$ given the past observations $\boldsymbol{t}$ is obtained from Equations (13), (17) and (18) and written as

$$
f^{*}\left(t_{a}^{*} \mid \boldsymbol{t}\right)=\int_{\theta} h\left(t_{a}^{*} \mid \theta, \boldsymbol{t}\right) \pi^{*}(\theta \mid \boldsymbol{t}) \mathrm{d} \theta, t_{a}^{*}>t_{r},
$$

where for $m \neq-1$,

$$
\begin{aligned}
h\left(t_{a}^{*} \mid \theta, t\right) \pi^{*}(\theta \mid t) \propto & C(\theta ; v) \exp [-D(\theta ; v)]\left[p_{1} f_{1}\left(t_{a}^{*}\right)+p_{2} f_{2}\left(t_{a}^{*}\right)\right] \\
& \times\left\{\prod_{i=s}^{r}\left[p_{1} R_{1}\left(t_{i}\right)+p_{2} R_{2}\left(t_{i}\right)\right]^{m}\left[p_{1} f_{1}\left(t_{i}\right)+p_{2} f_{2}\left(t_{i}\right)\right]\right\} \\
& \times \sum_{i=0}^{a-1} \sum_{\ell=0}^{s-1}\left\{\eta_{i, \ell}^{(a, s)}\left[p_{1} R_{1}\left(t_{s}\right)+p_{2} R_{2}\left(t_{s}\right)\right]^{\delta_{\ell}^{(s)}}\left[p_{1} R_{1}\left(t_{r}\right)+p_{2} R_{2}\left(t_{r}\right)\right]^{\delta_{i}^{(a)}}\right. \\
& \left.\times\left[p_{1} R_{1}\left(t_{a}^{*}\right)+p_{2} R_{2}\left(t_{a}^{*}\right)\right]^{\gamma_{r+a-i}-1}\right\}
\end{aligned}
$$

where

$$
\left.\begin{array}{rl}
\eta_{i, \ell}^{(a, s)} & =(-1)^{i+\ell}\left(\begin{array}{c}
a-1 \\
i
\end{array}\right)\left(\begin{array}{c}
s-1 \\
\ell
\end{array}\right), \\
\delta_{i}^{(a)} & =(a-i-1)(m+1), \\
\delta_{\ell}^{(s)} & =(s-\ell-1)(m+1) .
\end{array}\right\}
$$


Also, for $m=-1$,

$$
\begin{aligned}
h\left(t_{a}^{*} \mid \theta, \boldsymbol{t}\right) \pi^{*}(\theta \mid \boldsymbol{t}) \propto & C(\theta ; v) \exp [-D(\theta ; v)]\left[p_{1} f_{1}\left(t_{a}^{*}\right)+p_{2} f_{2}\left(t_{a}^{*}\right)\right] \\
& \times\left[p_{1} R_{1}\left(t_{a}^{*}\right)+p_{2} R_{2}\left(t_{a}^{*}\right)\right]^{k-1}\left(\ln \left[p_{1} R_{1}\left(t_{s}\right)+p_{2} R_{2}\left(t_{s}\right)\right]\right)^{s-1} \\
& \times\left\{\prod_{i=s}^{r} \frac{\sum_{j=1}^{2} p_{j} f_{j}\left(t_{i}\right)}{\sum_{j=1}^{2} p_{j} R_{j}\left(t_{i}\right)}\right\} \sum_{i=0}^{a-1} \omega_{i}^{(a)}\left(\ln \left[p_{1} R_{1}\left(t_{a}^{*}\right)+p_{2} R_{2}\left(t_{a}^{*}\right)\right]\right)^{i} \\
& \times\left(\ln \left[p_{1} R_{1}\left(t_{r}\right)+p_{2} R_{2}\left(t_{r}\right)\right]\right)^{a-i-1} .
\end{aligned}
$$

It then follows that the predictive survival function is given, for the $a^{\text {th }}$ future GOS, by

$$
P\left[T_{a}^{*}>v \mid \boldsymbol{t}\right]=\int_{v}^{\infty} f^{*}\left(t_{a}^{*} \mid \boldsymbol{t}\right) \mathrm{d} t_{a}^{*}, v>t_{r} .
$$

A $100 \tau \%$ BPI for $T_{a}^{*}$ is then given by

$$
P\left[L(\boldsymbol{t})<T_{a}^{*}<U(\boldsymbol{t})\right]=\tau,
$$

where $L(\boldsymbol{t})$ and $U(\boldsymbol{t})$ are obtained, respectively, by solving the following two equations

$$
\begin{aligned}
& P\left[T_{a}^{*}>L(\boldsymbol{t}) \mid \boldsymbol{t}\right]=\frac{1+\tau}{2}, \\
& P\left[T_{a}^{*}>U(\boldsymbol{t}) \mid \boldsymbol{t}\right]=\frac{1-\tau}{2} .
\end{aligned}
$$

\section{Two Sample Prediction}

Suppose that $T_{s ; n, m, k}, T_{s+1 ; n, m, k}, \cdots, T_{r ; n, m, k}, 1 \leq s<r \leq n$.

Be a doubly type II censored random sample drawn from a population whose CDF, $F(x)$ and PDF, $f(x)$ and let $Y_{1 ; N, M, K}, Y_{2 ; N, M, K}, \cdots, Y_{N ; N, M, K}, K \geq 1, M \geq-1$.

Be a second independent generalized ordered random sample (of size $N$ ) of future observations from the same distribution. Based on such a doubly type II censored sample, we wish to predict the future GOS $Y_{b} \equiv Y_{b ; N, M, K}, b=1,2, \cdots, N$, in the future sample of size $N$.

It was shown by [32] that the PDF of GOS $Y_{b}$ is in the form

$$
h_{Y_{b}}^{\star}(y \mid \theta) \propto\left\{\begin{array}{l}
f(y) \sum_{i=0}^{b-1} \omega_{i}^{(b)}[R(y)]^{\gamma_{b-i}^{\star}-1}, M \neq-1, \\
{[R(y)]^{K-1}[\ln R(y)]^{b-1} f(y), M=-1,}
\end{array}\right.
$$

where $\gamma_{i}^{\star}=K+(N-i)(M+1)$ and $\omega_{i}^{(b)}=(-1)^{i}\left(\begin{array}{c}b-1 \\ i\end{array}\right)$.

Substituting from Equations (1) and (5) in (25), we have

$$
h_{Y_{b}}^{\star}(y \mid \theta) \propto\left\{\begin{array}{l}
{\left[p_{1} f_{1}(y)+p_{2} f_{2}(y)\right] \sum_{i=0}^{b-1} \omega_{i}^{(b)}\left[p_{1} R_{1}(y)+p_{2} R_{2}(y)\right]^{\gamma_{b-i}^{\star}-1}, M \neq-1,} \\
{\left[p_{1} R_{1}(y)+p_{2} R_{2}(y)\right]^{K-1}\left(\ln \left[p_{1} R_{1}(y)+p_{2} R_{2}(y)\right]\right)^{b-1}\left[p_{1} f_{1}(y)+p_{2} f_{2}(y)\right], M=-1 .}
\end{array}\right.
$$

The predictive PDF of $Y_{b}, b=1,2, \cdots, N$, given the past observation $\mathrm{t}$ is obtained from Equations (14), (15) and (26), and written as

$$
f_{Y_{b}}^{\star}(y \mid \boldsymbol{t})=\int_{\theta} h_{Y_{b}}^{\star}(y \mid \theta) \pi^{*}(\theta \mid \boldsymbol{t}) \mathrm{d} \theta, \quad y>0,
$$

where for $m \neq-1, M \neq-1$, 


$$
\begin{aligned}
h_{Y_{b}}^{\star}(y \mid \theta) \pi^{*}(\theta \mid \boldsymbol{t}) \propto & C(\theta ; v) \exp [-D(\theta ; v)]\left\{\prod_{i=s}^{r}\left[p_{1} R_{1}\left(t_{i}\right)+p_{2} R_{2}\left(t_{i}\right)\right]^{m}\right. \\
& \left.\times\left[p_{1} f_{1}\left(t_{i}\right)+p_{2} f_{2}\left(t_{i}\right)\right]\right\}\left[p_{1} R_{1}\left(t_{r}\right)+p_{2} R_{2}\left(t_{r}\right)\right]^{\gamma_{r+1}}, \\
& \times\left[p_{1} f_{1}(y)+p_{2} f_{2}(y)\right] \sum_{i=0}^{b-1} \sum_{\ell=0}^{s-1}\left\{\eta_{i, \ell}^{(b, s)}\left[p_{1} R_{1}(y)+p_{2} R_{2}(y)\right]^{\gamma_{b-i}^{\star}-1}\right\}
\end{aligned}
$$

where

$$
\eta_{i, \ell}^{(b, s)}=(-1)^{i+\ell}\left(\begin{array}{c}
b-1 \\
i
\end{array}\right)\left(\begin{array}{c}
s-1 \\
\ell
\end{array}\right)
$$

Also for $m=-1, M=-1$,

$$
\begin{aligned}
h_{Y_{b}}^{\star}(y \mid \theta) \pi^{*}(\theta \mid \boldsymbol{t}) \propto & C(\theta ; v) \exp [-D(\theta ; v)]\left(\ln \left[p_{1} R_{1}(y)+p_{2} R_{2}(y)\right]\right)^{b-1} \\
& \times\left[p_{1} R_{1}(y)+p_{2} R_{2}(y)\right]^{K-1}\left[p_{1} f_{1}(y)+p_{2} f_{2}(y)\right] \\
& \times\left[p_{1} R_{1}\left(t_{r}\right)+p_{2} R_{2}\left(t_{r}\right)\right]^{k}\left(\ln \left[p_{1} R_{1}\left(t_{s}\right)+p_{2} R_{2}\left(t_{s}\right)\right]\right)^{s-1} . \\
& \times\left\{\prod_{i=s}^{r} \frac{\sum_{j=1}^{2} p_{j} f_{j}\left(t_{i}\right)}{\sum_{j=1}^{2} p_{j} R_{j}\left(t_{i}\right)}\right\}
\end{aligned}
$$

Bayesian prediction bounds for $Y_{b}, \quad b=1,2, \cdots, N$ are obtained by evaluating

$$
P\left[Y_{b}>v \mid \boldsymbol{t}\right]=\int_{v}^{\infty} f_{Y_{b}}^{\star}(y \mid \boldsymbol{t}) \mathrm{d} y, v>0 .
$$

A $100 \tau \%$ BPI for $Y_{b}$ is then given by

$$
P\left[L(\boldsymbol{t})<Y_{b}<U(\boldsymbol{t})\right]=\tau,
$$

where $L(\boldsymbol{t})$ and $U(\boldsymbol{t})$ are obtained, respectively, by solving the following two equations

$$
\begin{aligned}
& P\left[Y_{b}>L(\boldsymbol{t}) \mid \boldsymbol{t}\right]=\frac{1+\tau}{2}, \\
& P\left[Y_{b}>U(\boldsymbol{t}) \mid \boldsymbol{t}\right]=\frac{1-\tau}{2} .
\end{aligned}
$$

\section{Bayesian Prediction for the Future Median}

The median of $N$ observations, denoted by $\tilde{Y}_{N}$, is defined by

$$
\tilde{Y}_{N}=\left\{\begin{array}{l}
Y_{\varphi ; N, M, K}, N=2 \varphi-1 \\
\frac{1}{2}\left[Y_{\varphi ; N, M, K}+Y_{\varphi+1 ; N, M, K}\right], \quad N=2 \varphi
\end{array},\right.
$$

where $\varphi$ is a positive integer, $\varphi \geq 1$.

\subsection{The Case of Odd Future Sample Size}

The PDF of future median $\tilde{Y}_{N}$ takes the form (26) with $b=\varphi$ and $N=2 \varphi-1$.

Substituting $b=\varphi$ in Equation (27), we obtain the predictive PDF $f_{\tilde{Y}_{N}}^{\star}(y \mid \boldsymbol{t})$ of the median of $N=2 \varphi-1$ observations.

A $100 \tau \%$ BPI for $\tilde{Y}_{N}$ is then given by

$$
P\left[L(\boldsymbol{t})<\tilde{Y}_{N}<U(\boldsymbol{t})\right]=\tau,
$$


where $L(\boldsymbol{t})$ and $U(\boldsymbol{t})$ are obtained, respectively, by solving the following two equations

$$
\begin{aligned}
& P\left[\tilde{Y}_{N}>L(\boldsymbol{t}) \mid \boldsymbol{t}\right]=\frac{1+\tau}{2}, \\
& P\left[\tilde{Y}_{N}>U(\boldsymbol{t}) \mid \boldsymbol{t}\right]=\frac{1-\tau}{2},
\end{aligned}
$$

where, for $v>0, P\left[\tilde{Y}_{N}>v \mid \boldsymbol{t}\right]$ is predictive survival function, given by Equation (30) with $b=\varphi$ and $N=2 \varphi-1$.

\subsection{The Case of Even Future Sample Size}

The joint density function of two consecutive GOS $Y_{\varphi ; N, M, K} \equiv Y_{\varphi}$ and $Y_{\varphi+1 ; N, M, K} \equiv Y_{\varphi+1}$ is given by

$$
\hbar_{Y_{\varphi}, Y_{\varphi+1}}\left(y_{\varphi}, y_{\varphi+1} \mid \theta\right)=\left\{\begin{array}{l}
\frac{C_{\varphi}^{\star}}{(\varphi-1) !}\left[R\left(y_{\varphi}\right)\right]^{M}\left[h_{M}\left(F\left(y_{\varphi}\right)\right)-h_{M}(0)\right]^{\varphi-1} f\left(y_{\varphi}\right) \\
\quad \times\left[R\left(y_{\varphi+1}\right)\right]^{\gamma_{\varphi+1}^{\star}-1} f\left(y_{\varphi+1}\right), \quad M \neq-1 \\
\frac{K^{\varphi+1}}{(\varphi-1) !} H\left(y_{\varphi}\right) H\left(y_{\varphi+1}\right)\left[S\left(y_{\varphi}\right)\right]^{\varphi-1}\left[R\left(y_{\varphi+1}\right)\right]^{K}, \quad M=-1
\end{array},\right.
$$

where

$$
\left.\begin{array}{l}
C_{\varphi}^{\star}=\prod_{j=1}^{\varphi+1} \gamma_{j}^{\star}, \gamma_{j}^{\star}=K+(N-j)(M+1), \\
S(.)=-\ln (\bar{F}(.)),
\end{array}\right\}
$$

And

$$
h_{M}\left(F\left(y_{\varphi}\right)\right)= \begin{cases}\frac{-\left(\bar{F}\left(y_{\varphi}\right)\right)^{M+1}}{M+1}, & M \neq-1 \\ -\ln \left(\bar{F}\left(y_{\varphi}\right)\right), & M=-1\end{cases}
$$

Expanding $\left[h_{M}\left(F\left(y_{\varphi}\right)\right)-h_{M}(0)\right]^{\varphi-1}$ binomially and applying the transformation $\tilde{Y}_{N}=\frac{Y_{\varphi}+Y_{\varphi+1}}{2}$ and $Z=Y_{\varphi}$, the Jacobian of transformation is 2, we obtain

$$
\hbar_{z, \tilde{Y}_{N}}(z, y \mid \theta) \propto\left\{\begin{array}{l}
\sum_{j=0}^{\varphi-1} \omega_{j}^{(\varphi)}[R(z)]^{j(M+1)+M}[R(2 y-z)]^{\gamma_{\varphi+1}^{\star}-1} f(z) f(2 y-z), M \neq-1 \\
H(z) H(2 y-z)(\ln [R(z)])^{\varphi-1}[R(2 y-z)]^{K}, M=-1
\end{array} .\right.
$$

By substituting Equations (2), (4) and (5) in Equation (36) and integrating out $z$, yields the density function of $\tilde{Y}_{N}$, in the case of $M \neq-1$, as

$$
\begin{aligned}
\hbar_{\tilde{Y}_{N}}(y \mid \theta) \propto & \sum_{j=0}^{\varphi-1} \omega_{j}^{(\varphi)} \int_{0}^{y}\left[p_{1} R_{1}(z)+p_{2} R_{2}(z)\right]^{j(M+1)+M}\left[p_{1} R_{1}(2 y-z)+p_{2} R_{2}(2 y-z)\right]^{\gamma_{\varphi+1}^{*}-1} \\
& \times\left[p_{1} f_{1}(z)+p_{2} f_{2}(z)\right]\left[p_{1} f_{1}(2 y-z)+p_{2} f_{2}(2 y-z)\right] \mathrm{d} z, \quad y>0 .
\end{aligned}
$$

In the case $M=-1$, we have

$$
\begin{aligned}
\hbar_{\tilde{Y}_{N}}(y \mid \theta) \propto & \int_{0}^{y} \sum_{j=1}^{2} \frac{p_{j} f_{j}(z)}{p_{j} R_{j}(z)} \sum_{j=1}^{2} \frac{p_{j} f_{j}(2 y-z)}{p_{j} R_{j}(2 y-z)}\left(\ln \left[p_{1} R_{1}(z)+p_{2} R_{2}(z)\right]\right)^{\varphi-1} \\
& \times\left[p_{1} R_{1}(2 y-z)+p_{2} R_{2}(2 y-z)\right]^{K} \mathrm{~d} z, \quad y>0 .
\end{aligned}
$$


The predictive density function of the future median of $N=2 \varphi$ observations is given by

$$
f_{\tilde{Y}_{N}}^{\star}(y \mid \boldsymbol{t})=\int_{\theta} \hbar_{\tilde{Y}_{N}}(y \mid \theta) \pi^{*}(\theta \mid \boldsymbol{t}) \mathrm{d} \theta, \quad y>0,
$$

where $\pi^{*}(\theta \mid \boldsymbol{t})$ and $\hbar_{\tilde{Y}_{N}}(y \mid \theta)$ are given by Equations (13) and (37), (38), respectively. It then follows that the predictive survival function is given, for $\tilde{Y}_{N}$, by

$$
P\left[\tilde{Y}_{N}>v \mid \boldsymbol{t}\right]=\int_{v}^{\infty} f_{\tilde{Y}_{N}}^{\star}(y \mid t) \mathrm{d} y, v>0 .
$$

The lower and upper bound of $100 \tau \%$ BPI for $\tilde{Y}_{N}$ can be obtained by solving Equations (33) and (34), numerically.

\section{Example}

\section{Gompertz Components}

Suppose that, for $j=1,2$ and $t>0, \theta_{j}=1, \lambda_{j}(t)=\frac{1}{\alpha_{j}}\left[\mathrm{e}^{\alpha_{j} t}-1\right]$ so $\grave{\lambda}_{j}(t)=\mathrm{e}^{\alpha_{j} t}$.

In this case, the $j^{\text {th }}$ subpopulation is Gompertz distribution with parameter $\alpha_{j}>0$. Let $p, \alpha_{1}$ and $\alpha_{2}$ are independent random variables such that $p \sim \operatorname{Beta}\left(b_{1}, b_{2}\right)$ and for $j=1,2, \alpha_{j}$ to follow a left truncated exponential density with parameter $d_{j}\left(\operatorname{LTE}\left(d_{j}\right)\right)$, as used by [40]. A joint prior density function is then given by

$$
\pi(\theta ; v) \propto p_{1}^{b_{1}-1} p_{2}^{b_{2}-1} \exp \left[-\sum_{j=1}^{2} \frac{\left(\alpha_{j}-1\right)}{d_{j}}\right],
$$

where $0<p_{1}<1, \alpha_{j}>1,\left(b_{1}, b_{2}, d_{1}, d_{2}\right)>0$ and $p_{2}=1-p_{1}$.

\subsubsection{One Sample Prediction}

For $m \neq-1, m=-1$ substituting $\lambda_{j}(t)$, $\lambda_{j}(t)$.

And Equation (41) in Equation (22) and solving, numerically, Equations (23) and (24) we can obtain the lower and upper bounds of BPI.

\section{Special Cases}

1) Upper order statistics

The predictive PDF (19), when $m=0$ and $k=1$ becomes

$$
\begin{aligned}
f^{*}\left(t_{a}^{*} \mid \boldsymbol{t}\right)= & K_{1} \int_{0}^{1} \int_{0}^{\infty} \int_{0}^{\infty} p_{1}^{b_{1}-1} p_{2}^{b_{2}-1} \exp \left[-\sum_{j=1}^{2} \frac{\left(\alpha_{j}-1\right)}{d_{j}}\right] \\
& \times\left[p_{1} f_{1}\left(t_{a}^{*}\right)+p_{2} f_{2}\left(t_{a}^{*}\right)\right]\left\{\prod_{i=s}^{r}\left[p_{1} f_{1}\left(t_{i}\right)+p_{2} f_{2}\left(t_{i}\right)\right]\right\} \\
& \times \sum_{i=0}^{a-1} \sum_{\ell=0}^{s-1}\left\{\eta_{i, \ell}^{(a, s)}\left[p_{1} R_{1}\left(t_{s}\right)+p_{2} R_{2}\left(t_{s}\right)\right]^{s-\ell-1}\left[p_{1} R_{1}\left(t_{r}\right)+p_{2} R_{2}\left(t_{r}\right)\right]^{a-i-1},\right. \\
& \left.\times\left[p_{1} R_{1}\left(t_{a}^{*}\right)+p_{2} R_{2}\left(t_{a}^{*}\right)\right]^{n-r-a+i}\right\} \mathrm{d} \alpha_{1} \mathrm{~d} \alpha_{2} \mathrm{~d} p
\end{aligned}
$$

where

$$
K_{1}^{-1}=\int_{t_{r}}^{\infty} \int_{\theta} h\left(t_{a}^{*} \mid \theta, \boldsymbol{t}\right) \pi^{*}(\theta \mid \boldsymbol{t}) \mathrm{d} \theta \mathrm{d} t_{a}^{*} .
$$

Substituting from Equation (42) in Equation (22) and solving Equations (23) and (24), numerically, we can obtain the bounds of BPI.

2) Upper record values

When $m=-1, k=1\left(\gamma_{r}=1\right)$, the predictive PDF (19) becomes 


$$
\begin{aligned}
f^{*}\left(t_{a}^{*} \mid t\right)= & K_{2} \int_{0}^{1} \int_{0}^{\infty} \int_{0}^{\infty} p_{1}^{b_{1}-1} p_{2}^{b_{2}-1} \exp \left[-\sum_{j=1}^{2} \frac{\left(\alpha_{j}-1\right)}{d_{j}}\right]\left[p_{1} f_{1}\left(t_{a}^{*}\right)+p_{2} f_{2}\left(t_{a}^{*}\right)\right] \\
& \times\left(\ln \left[p_{1} R_{1}\left(t_{s}\right)+p_{2} R_{2}\left(t_{s}\right)\right]\right)^{s-1}\left\{\prod_{i=s}^{r} \frac{\sum_{j=1}^{2} p_{j} f_{j}\left(t_{i}\right)}{\sum_{j=1}^{2} p_{j} R_{j}\left(t_{i}\right)}\right\} \sum_{i=0}^{a-1} \omega_{i}^{(a)} \\
& \times\left(\ln \left[p_{1} R_{1}\left(t_{a}^{*}\right)+p_{2} R_{2}\left(t_{a}^{*}\right)\right]\right)^{i}\left(\ln \left[p_{1} R_{1}\left(t_{r}\right)+p_{2} R_{2}\left(t_{r}\right)\right]\right)^{a-i-1} \mathrm{~d} \alpha_{1} \mathrm{~d} \alpha_{2} \mathrm{~d} p
\end{aligned}
$$

where

$$
K_{2}^{-1}=\int_{t_{r}}^{\infty} \int_{\theta} h\left(t_{a}^{*} \mid \theta, \boldsymbol{t}\right) \pi^{*}(\theta \mid \boldsymbol{t}) \mathrm{d} \theta \mathrm{d} t_{a}^{*} .
$$

Substituting from Equation (43) in Equation (22) and solving Equations (23) and (24), numerically, we can obtain the bounds of BPI.

\subsubsection{Two Sample Prediction}

For $M \neq-1$ and $m \neq-1, M=-1$ and $m=-1$, substituting $\lambda_{j}(t), \grave{\lambda}_{j}(t)$ and Equation (41) in Equation (30) and solving, numerically, Equations (31) and (32) we can obtain the lower and upper bounds of BPI.

\section{Special Cases}

1) Upper order statistics

Substituting $M=m=0$ and $K=k=1$ in Equation (27), we have

$$
\begin{aligned}
f_{Y_{b}}^{\star}(y \mid t)= & B_{1} \int_{0}^{1} \int_{0}^{\infty} \int_{0}^{\infty} p_{1}^{b_{1}-1} p_{2}^{b_{2}-1} \exp \left[-\sum_{j=1}^{2} \frac{\left(\alpha_{j}-1\right)}{d_{j}}\right]\left\{\prod_{i=s}^{r}\left[p_{1} f_{1}\left(t_{i}\right)+p_{2} f_{2}\left(t_{i}\right)\right]\right\} \\
& \times\left[p_{1} R_{1}\left(t_{r}\right)+p_{2} R_{2}\left(t_{r}\right)\right]^{n-r}\left[p_{1} f_{1}(y)+p_{2} f_{2}(y)\right] \sum_{i=0}^{b-1} \sum_{\ell=0}^{s-1}\left\{\eta_{i, \ell}^{(b, s)},\right. \\
& \left.\times\left[p_{1} R_{1}\left(t_{s}\right)+p_{2} R_{2}\left(t_{s}\right)\right]^{s-\ell-1}\left[p_{1} R_{1}(y)+p_{2} R_{2}(y)\right]^{N-b+i}\right\} \mathrm{d} \alpha_{1} \mathrm{~d} \alpha_{2} \mathrm{~d} p
\end{aligned}
$$

where

$$
B_{1}^{-1}=\int_{0}^{\infty} \int_{\theta} h_{Y_{b}}^{\star}(y \mid \theta) \pi^{*}(\theta \mid \boldsymbol{t}) \mathrm{d} \theta \mathrm{d} y .
$$

To obtain $100 \tau \%$ BPI for $Y_{b}, b=1,2, \cdots, N$, we solve Equations (31) and (32), numerically.

2) Upper record values

In Equation (27), by putting $K=k=1$, the predictive PDF of $Y_{b}$ takes the form

$$
\begin{aligned}
f_{Y_{b}}^{\star}(y \mid \boldsymbol{t})= & B_{2} \int_{0}^{1} \int_{0}^{\infty} \int_{0}^{\infty} p_{1}^{b_{1}-1} p_{2}^{b_{2}-1} \exp \left[-\sum_{j=1}^{2} \frac{\left(\alpha_{j}-1\right)}{d_{j}}\right]\left(\ln \left[p_{1} R_{1}(y)+p_{2} R_{2}(y)\right]\right)^{b-1}, \\
& \times\left[p_{1} f_{1}(y)+p_{2} f_{2}(y)\right]\left[p_{1} R_{1}\left(t_{r}\right)+p_{2} R_{2}\left(t_{r}\right)\right] \\
& \times\left(\ln \left[p_{1} R_{1}\left(t_{s}\right)+p_{2} R_{2}\left(t_{s}\right)\right]\right)^{s-1}\left\{\prod_{i=s}^{r} \frac{\sum_{j=1}^{2} p_{j} f_{j}\left(t_{i}\right)}{\sum_{j=1}^{2} p_{j} R_{j}\left(t_{i}\right)}\right\} \mathrm{d} \alpha_{1} \mathrm{~d} \alpha_{2} \mathrm{~d} p
\end{aligned}
$$

where

$$
B_{2}^{-1}=\int_{0}^{\infty} \int_{\theta} h_{Y_{b}}^{\star}(y \mid \theta) \pi^{*}(\theta \mid \boldsymbol{t}) \mathrm{d} \theta \mathrm{d} y .
$$

Substituting from Equation (45) in Equation (30) and solving Equations (31) and (32), numerically, we can obtain the bounds of BPI.

\subsubsection{Prediction for the Future Median (the Case of Odd $N$ )}

\section{Special Cases}


1) Upper order statistics

Substituting $\lambda_{j}(t), \grave{\lambda}_{j}(t), C(\theta ; v)$ and $D(\theta ; v)$ in Equation (27) with $b=\varphi$ and $N=2 \varphi-1$ and by putting $M=m=0$ and $K=k=1$, we have

$$
\begin{aligned}
f_{\tilde{Y}_{N}}^{\star}(y \mid \boldsymbol{t})= & B_{1}^{\star} \int_{0}^{1} \int_{0}^{\infty} \int_{0}^{\infty} p_{1}^{b_{1}-1} p_{2}^{b_{2}-1} \exp \left[-\sum_{j=1}^{2} \frac{\left(\alpha_{j}-1\right)}{d_{j}}\right] \\
& \times\left\{\prod_{i=s}^{r}\left[p_{1} f_{1}\left(t_{i}\right)+p_{2} f_{2}\left(t_{i}\right)\right]\right\}\left[p_{1} R_{1}\left(t_{r}\right)+p_{2} R_{2}\left(t_{r}\right)\right]^{n-r}, \\
& \times\left[p_{1} f_{1}(y)+p_{2} f_{2}(y)\right] \sum_{i=0}^{\varphi-1} \sum_{\ell=0}\left\{\eta_{i, \ell}^{(\varphi, s)}\left[p_{1} R_{1}\left(t_{s}\right)+p_{2} R_{2}\left(t_{s}\right)\right]^{s-\ell-1}\right. \\
& \left.\times\left[p_{1} R_{1}(y)+p_{2} R_{2}(y)\right]^{N-\varphi+i}\right\} \mathrm{d} \alpha_{1} \mathrm{~d} \alpha_{2} \mathrm{~d} p
\end{aligned}
$$

where

$$
B_{1}^{\star-1}=\int_{0}^{\infty} \int_{\theta} h_{\tilde{Y}_{N}}^{\star}(y \mid \theta) \pi^{*}(\theta \mid \boldsymbol{t}) \mathrm{d} \theta \mathrm{d} y .
$$

To obtain $100 \tau \%$ BPI for $\tilde{Y}_{N}$, we solve Equations (33) and (34), numerically.

2) Upper record values

The predictive PDF (27), when $K=k=1$, becomes

$$
\begin{aligned}
f_{\tilde{Y}_{N}}^{\star}(y \mid t)= & B_{2}^{\star} \int_{0}^{1} \int_{0}^{\infty} \int_{0}^{\infty} p_{1}^{b_{1}-1} p_{2}^{b_{2}-1} \exp \left[-\sum_{j=1}^{2} \frac{\left(\alpha_{j}-1\right)}{d_{j}}\right]\left(\ln \left[p_{1} R_{1}(y)+p_{2} R_{2}(y)\right]\right)^{\varphi-1}, \\
& \times\left[p_{1} f_{1}(y)+p_{2} f_{2}(y)\right]\left[p_{1} R_{1}\left(t_{r}\right)+p_{2} R_{2}\left(t_{r}\right)\right] \\
& \times\left(\ln \left[p_{1} R_{1}\left(t_{s}\right)+p_{2} R_{2}\left(t_{s}\right)\right]\right)^{s-1} \times\left\{\prod_{i=s}^{r} \frac{\sum_{j=1}^{2} p_{j} f_{j}\left(t_{i}\right)}{\sum_{j=1}^{2} p_{j} R_{j}\left(t_{i}\right)}\right\} \mathrm{d} \alpha_{1} \mathrm{~d} \alpha_{2} \mathrm{~d} p
\end{aligned}
$$

where

$$
B_{2}^{\star-1}=\int_{0}^{\infty} \int_{\theta} h_{\tilde{Y}_{N}}^{\star}(y \mid \theta) \pi^{*}(\theta \mid \boldsymbol{t}) \mathrm{d} \theta \mathrm{d} y .
$$

To obtain $100 \tau \%$ BPI for $\tilde{Y}_{N}$, we solve Equations (33) and (34), numerically.

\subsubsection{Prediction for the Future Median (the Case of Even $N$ )}

\section{Special Cases}

1) Upper order statistics

The predictive PDF and survival function of $\tilde{Y}_{N}$ can be obtained by substituting $M=m=0$ and $K=k=1$ in Equations (39) and (40), respectively.

2) Upper record values

The predictive PDF and survival function of $\tilde{Y}_{N}$ can be obtained by substituting $K=k=1$ in Equations (39) and (40), respectively.

To obtain $100 \tau \%$ BPI for future median of ordinary order statistics or ordinary upper record values.

We solve Equations (33) and (34), numerically.

\section{Numerical Computations}

In this section, 95\% BPI for future observations from a mixture of two $\operatorname{Gomp}\left(\alpha_{j}\right), j=1,2$, components are obtained by considering one sample and two sample schemes.

\subsection{One Sample Prediction}

In this subsection, we compute 95\% BPI for $T_{a}^{*}, a=1,2$, in the two cases ordinary order statistics and ordi- 
nary upper record values according to the following steps:

1) For a given values of the prior parameters $\left(b_{1}, b_{2}\right)$ generate a random value $p$ from the $\operatorname{Beta}\left(b_{1}, b_{2}\right)$ distribution.

2) For a given values of the prior parameters $d_{j}$, for $j=1,2$, generate a random value $\alpha_{j}$ from the $\operatorname{LTE}\left(d_{j}\right)$ distribution.

3) Using the generated values of $p, \alpha_{1}$ and $\alpha_{2}$, we generate a random sample from a mixture of two $\operatorname{Gomp}\left(\alpha_{j}\right)$ components, $j=1,2$, as follows:

- generate two observations $u_{1}, u_{2}$ from $\operatorname{Uniform}(0,1)$;

- if $u_{1} \leq p$, then $t=\frac{1}{\alpha_{1}} \log \left[1-\alpha_{1} \log \left(1-u_{2}\right)\right]$, otherwise $t=\frac{1}{\alpha_{2}} \log \left[1-\alpha_{2} \log \left(1-u_{2}\right)\right]$;

- repeat above steps $n$ times to get a sample of size $n$;

- the sample obtained in above steps is ordered.

4) Using the generated values of $p, \alpha_{1}$ and $\alpha_{2}$, we generate upper record values of size $n=12$ from a mixture of two $\operatorname{Gomp}\left(\alpha_{j}\right), j=1,2$, components.

5) The 95\% BPI for the future observations are obtained by solving numerically, Equations (23) and (24) with $\tau=0.95$. Different sample size $n$ and the censored size are considered.

\subsection{Two Sample Prediction}

In this subsection, we compute 95\% BPI for two sample prediction in the two cases ordinary order statistics and ordinary upper record values according to the following steps:

1) For a given values of the prior parameters $\left(b_{1}, b_{2}\right)$ generate a random value $p$ from the $\operatorname{Beta}\left(b_{1}, b_{2}\right)$ distribution.

2) For a given values of the prior parameters $d_{j}$, for $j=1$,2, generate a random value $\alpha_{j}$ from the $\operatorname{LTE}\left(d_{j}\right)$ distribution.

3) Using the generated values of $p, \alpha_{1}$ and $\alpha_{2}$, we generate a doubly type II sample from a mixture of two $\operatorname{Gomp}\left(\alpha_{j}\right), j=1,2$, components.

4) The $95 \%$ BPI for the observations from a future independent sample of size $N$ are obtained by solving numerically, Equations (31) and (32) with $\tau=0.95$.

5) Generate 10,000 samples each of size $N$ from a mixture of two $\operatorname{Gomp}\left(\alpha_{j}\right)$ components, then calculate the coverage percentage of $Y_{b}$.

6) Different sample sizes $n$ and $N$ are considered.

\subsection{Prediction for the Future Median}

In this subsection, 95\% BPI for the median of $N$ future observations are obtained when the underlying population distribution is a mixture of two Gompertz components in the two cases ordinary order statistics and ordinary upper record values according to the following steps:

1) For a given values of the prior parameters $\left(b_{1}, b_{2}\right)$ generate a random value $p$ from the $\operatorname{Beta}\left(b_{1}, b_{2}\right)$ distribution.

2) For a given values of the prior parameters $d_{j}$, for $j=1,2$, generate a random value $\alpha_{j}$ from the $\operatorname{LTE}\left(d_{j}\right)$ distribution.

3) Using the generated values of $p, \alpha_{1}$ and $\alpha_{2}$, we generate a doubly type II sample from a mixture of two $\operatorname{Gomp}\left(\alpha_{j}\right), j=1,2$, components.

4) The $95 \%$ BPI for the median of $N$ of future observations are obtained by solving numerically, Equations (33) and (34) with $\tau=0.95$ for different values of $N$, when $N=2 \varphi-1$ is odd and $N=2 \varphi$ is even.

5) Generate 10,000 samples each of size $N$ from a mixture of two $\operatorname{Gomp}\left(\alpha_{j}\right)$ components, then calculate the coverage percentage of $\tilde{Y}_{N}$.

6) The prediction are conducted on the basis of a doubly type II censored samples and type II censored samples.

The computational (our) results were computed by using Mathematica 6.0. When the prior parameters chosen as $b_{1}=1.5, b_{2}=2, d_{1}=1, d_{2}=2$ which yield the generated values of $p=0.516065, \alpha_{1}=1.46186$, $\alpha_{2}=3.1847$. In Tables 1-4, 95\% BPI for future observations are computed in case of the one and two 
Table 1. 95\% BPI for future order statistics $T_{a}^{*}, a=1,2$, when $b_{1}=1.5, b_{2}=2, d_{1}=1, d_{2}=2$ and the generated parameters $\left(p=0.516065, \alpha_{1}=1.46186, \alpha_{2}=3.1847\right)$.

\begin{tabular}{cccccc}
\hline Case & & \multicolumn{2}{c}{$S=1$} & & $S=2$ \\
$(n, r)$ & $T_{a}^{*}$ & $(L, U)$ & Length & $(L, U)$ & Length \\
\cline { 3 - 6 }$(10,7)$ & $T_{1}^{*}$ & $(0.562965,0.744602)$ & 0.181638 & $(0.443015,0.618514)$ & 0.175499 \\
& $T_{2}^{*}$ & $(0.748781,1.53094)$ & 0.782164 & $(0.569291,1.30527)$ & 0.735977 \\
$(15,10)$ & $T_{1}^{*}$ & $(0.47374,0.548465)$ & 0.0747253 & $(0.40578,0.480169)$ & 0.0743882 \\
& $T_{2}^{*}$ & $(0.587001,0.901358)$ & 0.314357 & $(0.494304,0.804901)$ & 0.310597 \\
$(20,15)$ & $T_{1}^{*}$ & $(0.719253,0.774191)$ & 0.0549385 & $(0.601514,0.65858)$ & 0.0570667 \\
& $T_{2}^{*}$ & $(0.866788,1.14337)$ & 0.276584 & $(0.740253,1.01961)$ & 0.279359 \\
$(50,35)$ & $T_{1}^{*}$ & $(0.789649,0.797004)$ & 0.00735491 & $(0.555976,0.563516)$ & 0.00754019 \\
& $T_{2}^{*}$ & $(0.791368,0.883652)$ & 0.0922842 & $(0.559453,0.816295)$ & 0.256842 \\
\hline
\end{tabular}

Table 2. 95\% BPI for the future upper record values $T_{a}^{*}, a=1,2$, when $b_{1}=1.5, b_{2}=2, d_{1}=1, d_{2}=2$ and the generated parameters $\left(p=0.516065, \alpha_{1}=1.46186, \alpha_{2}=3.1847\right)$.

\begin{tabular}{cccccc}
\hline$r$ & & \multicolumn{2}{c}{$S=1$} & & $S=2$ \\
\cline { 3 - 6 } & $T_{a}^{*}$ & $(L, U)$ & Length & $(L, U)$ & 1.02492 \\
\hline \multirow{2}{*}{5} & $T_{1}^{*}$ & $(1.47048,2.71682)$ & 1.24633 & $(0.783431,1.80835)$ & 1.73175 \\
& $T_{2}^{*}$ & $(1.50643,3.48872)$ & 1.98229 & $(0.803645,2.5354)$ & 0.643359 \\
& $T_{1}^{*}$ & $(1.38189,1.94658)$ & 0.564687 & $(1.80196,2.44531)$ & 0.998218 \\
& $T_{2}^{*}$ & $(1.36459,2.32526)$ & 0.960663 & $(1.8222,2.82042)$ & 0.58877 \\
& $T_{1}^{*}$ & $(1.93128,2.45302)$ & 0.52174 & $(1.90637,2.49514)$ & 0.906915 \\
\hline
\end{tabular}

Table 3. 95\% BPI and PC for the future order statistics $Y_{b}, b=1,2$, when $b_{1}=1.5, b_{2}=2, d_{1}=1, d_{2}=2$ and the generated parameters $\left(p=0.516065, \alpha_{1}=1.46186, \alpha_{2}=3.1847\right)$.

\begin{tabular}{|c|c|c|c|c|c|}
\hline \multirow{2}{*}{$\begin{array}{c}N \\
(n, r)\end{array}$} & \multirow{2}{*}{$Y_{b}$} & \multicolumn{2}{|l|}{$S=1$} & \multicolumn{2}{|l|}{$S=2$} \\
\hline & & $(L, U)$ Length & $P C$ & $(L, U)$ Length & $P C$ \\
\hline \multirow{2}{*}{$\begin{array}{c}10 \\
(20,15)\end{array}$} & $Y_{1}$ & $\begin{array}{c}(0.00253099,0.357705) \\
0.355174\end{array}$ & 97.13 & $\begin{array}{c}(0.00253088,0.354125) \\
0.351594\end{array}$ & 97.32 \\
\hline & $Y_{2}$ & $\begin{array}{c}(0.0249745,0.561048) \\
0.536074\end{array}$ & 97.50 & $\begin{array}{c}(0.0250174,0.552829) \\
0.527811\end{array}$ & 97.55 \\
\hline \multirow{2}{*}{$\begin{array}{c}10 \\
(20,18)\end{array}$} & $Y_{1}$ & $\begin{array}{c}(0.00253086,0.352991) \\
0.35046\end{array}$ & 96.72 & $\begin{array}{c}(0.00253091,0.353966) \\
0.351435\end{array}$ & 96.99 \\
\hline & $Y_{2}$ & $\begin{array}{c}(0.0250486,0.550266) \\
0.525218\end{array}$ & 97.33 & $\begin{array}{c}(0.0250276,0.552571) \\
0.527543\end{array}$ & 97.29 \\
\hline \multirow{2}{*}{$\begin{array}{c}15 \\
(30,22)\end{array}$} & $Y_{1}$ & $\begin{array}{c}(0.00168778,0.244183) \\
0.242495\end{array}$ & 96.79 & $\begin{array}{c}(0.00168788,0.244617) \\
0.242929\end{array}$ & 96.53 \\
\hline & $Y_{2}$ & $\begin{array}{c}(0.018541,0.382363) \\
0.363821\end{array}$ & 96.59 & $\begin{array}{c}(0.0194943,0.384252) \\
0.364758\end{array}$ & 96.26 \\
\hline \multirow{2}{*}{$\begin{array}{c}15 \\
(30,27)\end{array}$} & $Y_{1}$ & $\begin{array}{c}(0.00168747,0.241649) \\
0.239962\end{array}$ & 96.56 & $\begin{array}{c}(0.0016876,0.243342) \\
0.241654\end{array}$ & 96.76 \\
\hline & $Y_{2}$ & $\begin{array}{c}(0.0163242,0.374301) \\
0.357976\end{array}$ & 97.29 & $\begin{array}{c}(0.0172091,0.379132) \\
0.361923\end{array}$ & 96.92 \\
\hline
\end{tabular}


sample predictions, respectively. In Table 5 and Table 6, 95\% BPI for the medians of future samples with odd or even sizes are computed. Our results are specialized to ordinary order statistics and ordinary upper record values.

Table 4. 95\% BPI and $P C$ for future ordinary upper record values $Y_{b}, b=1,2$, when $b_{1}=1.5, b_{2}=2, d_{1}=1, d_{2}=2$ and the generated parameters $\left(p=0.516065, \alpha_{1}=1.46186, \alpha_{2}=3.1847\right)$.

\begin{tabular}{cccccc}
\hline $\begin{array}{c}\text { Case } N \\
(n, r)\end{array}$ & $Y_{b}$ & \multicolumn{2}{c}{$S=1$} & & \multicolumn{2}{c}{$S=2$} \\
\cline { 3 - 6 } & & $(L, U)$ Length & $P C$ & $(L, U)$ Length & $P C$ \\
\hline 6 & $Y_{1}$ & $(0.0244019,1.26507) 1.24067$ & 97.19 & $(0.0242337,1.26247) 1.23824$ & 97.20 \\
$(8,5)$ & $Y_{2}$ & $(0.184594,1.79401) 1.60942$ & 97.02 & $(0.177304,1.8683) 1.69099$ & 97.44 \\
6 & $Y_{1}$ & $(0.0243355,1.17383) 1.14949$ & 96.89 & $(0.0245445,1.25834) 1.23379$ & 96.91 \\
$(8,7)$ & $Y_{2}$ & $(0.179104,1.49161) 1.31251$ & 97.36 & $(0.188427,1.62202) 1.43359$ & 97.37 \\
8 & $Y_{1}$ & $(0.0240923,1.1295) 1.10541$ & 96.68 & $(0.0244941,1.20562) 1.18112$ & 96.92 \\
$(10,7)$ & $Y_{2}$ & $(0.169789,1.47476) 1.30497$ & 96.85 & $(0.187398,1.59348) 1.40609$ & 96.03 \\
$(10,9)$ & $Y_{1}$ & $(0.0239792,1.08241) 1.05843$ & 96.36 & $(0.0237464,1.11903) 1.09529$ & 96.75 \\
\hline
\end{tabular}

Table 5. (Ordinary order statistics) $95 \%$ BPI and $P C$ for future median $\tilde{Y}_{N}$ when $N=2 \varphi-1$ is odd or $2 \varphi$, is even and $b_{1}=1.5, b_{2}=2, d_{1}=1, d_{2}=2$ and the generated parameters $\left(p=0.516065, \alpha_{1}=1.46186, \alpha_{2}=3.1847\right)$.

\begin{tabular}{cccccc}
\hline $\begin{array}{c}n=30 \\
r\end{array}$ & $\tilde{Y}_{N}$ & \multicolumn{2}{c}{$S=1$} & & \multicolumn{2}{c}{$S=2$} \\
\cline { 3 - 7 } & & $(L, U)$ Length & $P C$ & $(L, U)$ Length & $P C$ \\
\hline \multirow{2}{*}{18} & $\tilde{Y}_{5}$ & $(0.150064,1.7981) 1.64804$ & 96.70 & $(0.149628,1.81497) 1.66534$ & 96.55 \\
& $\tilde{Y}_{4}$ & $(0.15732,1.87535) 1.71803$ & 86.29 & $(0.156637,1.89352) 1.73689$ & 85.81 \\
& $\tilde{Y}_{5}$ & $(0.150599,1.78292) 1.63232$ & 96.50 & $(0.150879,1.77517) 1.62429$ & 96.70 \\
& $\tilde{Y}_{4}$ & $(0.158553,1.85921) 1.70066$ & 85.77 & $(0.159278,1.85101) 1.69173$ & 85.63 \\
& $\tilde{Y}_{5}$ & $(0.151131,1.76607) 1.61494$ & 96.39 & $(0.15093,1.77256) 1.62163$ & 96.69 \\
& $\tilde{Y}_{4}$ & $(0.160396,1.84172) 1.68132$ & 85.25 & $(0.159707,1.8485) 1.68879$ & 85.26 \\
\hline
\end{tabular}

Table 6. (Ordinary upper record values) $95 \% B P I$ and $P C$ for future median $\tilde{Y}_{N}$ when $N=2 \varphi-1$ is odd or $2 \varphi$, is even and $b_{1}=1.5, b_{2}=2, d_{1}=1, d_{2}=2$ and the generated parameters $\left(p=0.516065, \alpha_{1}=1.46186, \alpha_{2}=3.1847\right)$.

\begin{tabular}{|c|c|c|c|c|c|}
\hline \multirow{2}{*}{$\begin{array}{c}n=10 \\
r\end{array}$} & \multirow{2}{*}{$\tilde{Y}_{N}$} & \multicolumn{2}{|l|}{$S=1$} & \multicolumn{2}{|l|}{$S=2$} \\
\hline & & $(L, U)$ Length & $P C$ & $(L, U)$ Length & $P C$ \\
\hline \multirow{2}{*}{5} & $\tilde{Y}_{3}$ & $(0.160855,2.18738) 2.02653$ & 98.29 & $(0.145888,2.22403) 2.07814$ & 98.77 \\
\hline & $\tilde{Y}_{2}$ & $(0.133759,1.70622) 1.57246$ & 84.56 & $(0.148567,1.75259) 1.60402$ & 83.42 \\
\hline \multirow{2}{*}{7} & $\tilde{Y}_{3}$ & $(0.170573,2.00065) 1.83008$ & 98.14 & $(0.148896,1.98626) 1.83736$ & 98.66 \\
\hline & $\tilde{Y}_{2}$ & $(0.142946,1.5994) 1.45645$ & 84.74 & $(0.140236,1.60923) 1.469$ & 84.34 \\
\hline \multirow{2}{*}{9} & $\tilde{Y}_{3}$ & $(0.200611,1.52718) 1.32657$ & 96.87 & $(0.196739,1.51186) 1.31512$ & 96.75 \\
\hline & $\tilde{Y}_{2}$ & $(0.116557,1.29989) 1.18334$ & 86.34 & $(0.118077,1.27117) 1.1531$ & 86.46 \\
\hline
\end{tabular}




\subsection{Conclusions}

1) Bayes prediction intervals for future observations are obtained using a one-sample and two-sample schemes based on a finite mixture of two Gompertz components model. Our results are specialized to ordinary order statistics and ordinary upper record values.

2) Bayesian prediction intervals for the medians of future samples with odd or even sizes are obtained based on a finite mixture of two Gompertz components model. Our results are specialized to ordinary order statistics and ordinary upper record values.

3) It is evident from all tables that the lengths of the BPI decrease as the sample size increase.

4) In general, if the sample size $n$ and censored size $r$ are fixed the lengths of the BPI increase by increasing $s$.

5) For fixed sample size $n$, censored size $r$ and $s$, the lengths of the BPI increase by increasing $a$ or $b$.

6) The percentage coverage improves by the use of a large number of observed values.

\section{References}

[1] Geisser, S. (1993) Predictive Inference: An Introduction. Chapman and Hall, London. http://dx.doi.org/10.1007/978-1-4899-4467-2

[2] Aitchison, J. and Dunsmore, I.R. (1975) Statistical Prediction Analysis. Cambridge University Press, Cambridge. http://dx.doi.org/10.1017/CBO9780511569647

[3] AL-Hussaini, E.K. (1999) Prediction, Observables from a General Class of Distributions. Journal of Statistical Planning and Inference, 79, 79-91. http://dx.doi.org/10.1016/S0378-3758(98)00228-6

[4] AL-Hussaini, E.K. (1999) Bayesian Prediction under a Mixture of Two Exponential Components Model Based on Type I Censoring. Journal of Applied Statistical Science, 8, 173-185.

[5] AL-Hussaini, E.K. (2001) On Bayesian Prediction of Future Median. Communications in Statistics-Theory and Methods, 30, 1395-1410. http://dx.doi.org/10.1081/STA-100104752

[6] AL-Hussaini, E.K., Nigm, A.M. and Jaheen, Z.F. (2001) Bayesian Prediction Based on Finite Mixtures of Lomax Components Model and Type I Censoring. Statistics, 35, 259-268. http://dx.doi.org/10.1080/02331880108802735

[7] AL-Hussaini, E.K. (2003) Bayesian Predictive Density of Order Statistics Based on Finite Mixture Models. Journal of Statistical Planning and Inference, 113, 15-24. http://dx.doi.org/10.1016/S0378-3758(01)00297-X

[8] AL-Hussaini, E.K. and Ahmad, A.A. (2003) On Bayesian Interval Prediction of Future Records. Test, 12, 79-99. http://dx.doi.org/10.1007/BF02595812

[9] Jaheen, Z.F. (2003) Bayesian Prediction under a Mixture of Two-Component Gompertz Lifetime Model. Test, 12, 413426. http://dx.doi.org/10.1007/BF02595722

[10] AL-Hussaini, E.K. and AL-Jarallah, R.A. (2006) Bayes Inference under a Finite Mixture of Two Compound Gompertz Components Model. Cairo University, Giza.

[11] AL-Hussaini, E.K. and Osman, M.I. (1997) On the Median of a Finite Mixture. Journal of Statistical Computation and Simulation, 58, 121-144. http://dx.doi.org/10.1080/00949659708811826

[12] AL-Hussaini, E.K. and Jaheen, Z.F. (1999) Parametric Prediction Bounds for the Future Median of the Exponential Distribution. Statistics: A Journal of Theoretical and Applied Statistics, 32, 267-275. http://dx.doi.org/10.1080/02331889908802667

[13] Kamps, U. (1995) A Concept of Generalized Order Statistics. Journal of Statistical Planning and Inference, 48, 1-23. http://dx.doi.org/10.1016/0378-3758(94)00147-N

[14] Ahsanullah, M. (1996) Generalized Order Statistics from Two Parameter Uniform Distribution. Communications in Statistics: Theory and Methods, 25, 2311-2318. http://dx.doi.org/10.1080/03610929608831840

[15] Ahsanullah, M. (2000) Generalized Order Statistics from Exponential Distribution. Journal of Statistical Planning and Inference, 85, 85-91. http://dx.doi.org/10.1016/S0378-3758(99)00068-3

[16] Kamps, U. and Gather, U. (1997) Characteristic Property of Generalized Order Statistics for Exponential Distributions. Applicationes Mathematicae, 24, 383-391.

[17] Cramer, E. and Kamps, U. (2000) Relations for Expectations of Functions of Generalized Order Statistics. Journal of Statistical Planning and Inference, 89, 79-89. http://dx.doi.org/10.1016/S0378-3758(00)00074-4

[18] Cramer, E. and Kamps, U. (2001) On Distributions of Generalized Order Statistics. Statistics: A Journal of Theoretical and Applied Statistics, 35, 269-280. http://dx.doi.org/10.1080/02331880108802736

[19] Habibullah, M. and Ahsanullah, M. (2000) Estimation of Parameters of a Pareto Distribution by Generalized Order 
Statistics. Communications in Statistics: Theory and Methods, 29, 1597-1609. http://dx.doi.org/10.1080/03610920008832567

[20] AL-Hussaini, E.K. and Ahmad, A.A. (2003) On Bayesian Interval Prediction of Future Records. Test, 12, 79-99. http://dx.doi.org/10.1007/BF02595812

[21] AL-Hussaini, E.K. and Ahmad, A.A. (2003) On Bayesian Predictive Distributions of Generalized Order Statistics. Metrika, 57, 165-176. http://dx.doi.org/10.1007/s001840200207

[22] AL-Hussaini, E.K. (2004) Generalized Order Statistics: Prospective and Applications. Journal of Applied Statistical Science, 13, 59-85.

[23] Jaheen, Z.F. (2002) On Bayesian Prediction of Generalized Order Statistics. Journal of Statistical Theory and Applications, 1, 191-204.

[24] Jaheen, Z.F. (2005) Estimation Based on Generalized Order Statistics from the Burr Model. Communications in Statistics: Theory and Methods, 34, 785-794. http://dx.doi.org/10.1081/STA-200054408

[25] Ahmad, A.A. (2007) Relations for Single and Product Moments of Generalized Order Statistics from Doubly Truncated Burr Type XII Distribution. Journal of the Egyptian Mathematical Society, 15, 117-128.

[26] Ahmad, A.A. (2008) Single and Product Moments of Generalized Order Statistics from Linear Exponential Distribution. Communications in Statistics: Theory and Methods, 37, 1162-1172. http://dx.doi.org/10.1080/03610920701713344

[27] Ahmad, A.A. (2011) On Bayesian Interval Prediction of Future Generalized Order Statistics Using Doubly Censoring. Statistics: A Journal of Theoretical and Applied Statistics, 45, 413-425. http://dx.doi.org/10.1080/02331881003650123

[28] Ateya, S.F. and Ahmad, A.A. (2011) Inferences Based on Generalized Order Statistics under Truncated Type I Generalized Logistic Distribution. Statistics: A Journal of Theoretical and Applied Statistics, 45, 389-402. http://dx.doi.org/10.1080/02331881003650149

[29] Abu-Shal, T.A. and AL-Zaydi, A.M. (2012) Estimation Based on Generalized Order Statistics from a Mixture of Two Rayleigh Distributions. International Journal of Statistics and Probability, 1, 79-90.

[30] Abu-Shal, T.A. and AL-Zaydi, A.M. (2012) Bayesian Prediction Based on Generalized Order Statistics from a Mixture of Two Exponentiated Weibull Distribution via MCMC Simulation. International Journal of Statistics and Probability, 1, 20-34.

[31] Abu-Shal, T.A. and AL-Zaydi, A.M. (2012) Prediction Based on Generalized Order Statistics from a Mixture of Rayleigh Distributions Using MCMC Algorithm. Open Journal of Statistics, 2, 356-367. http://dx.doi.org/10.4236/ojs.2012.23044

[32] Abu-Shal, T.A. (2013) On Bayesian Prediction of Future Median Generalized Order Statistics Using Doubly Censored Data from Type-I Generalized Logistic Model. Journal of Statistical and Econometric Methods, 2, 61-79.

[33] Ahmad, A.A. and AL-Zaydi, A.M. (2013) Inferences under a Class of Finite Mixture Distributions Based on Generalized Order Statistics. Open Journal of Statistics, 3, 231-244. http://dx.doi.org/10.4236/ojs.2013.34027

[34] Everitt, B.S. and Hand, D.J. (1981) Finite Mixture Distributions. Springer Netherlands, Dordrecht. http://dx.doi.org/10.1007/978-94-009-5897-5

[35] Titterington, D.M., Smith, A.F.M. and Makov, U.E. (1985) Statistical Analysis of Finite Mixture Distributions. John Wiley and Sons, New York.

[36] McLachlan, G.J. and Basford, K.E. (1988) Mixture Models: Inferences and Applications to Clustering. Marcel Dekker, New York.

[37] Teicher, H. (1963) Identifiability of Finite Mixtures. The Annals of Mathematical Statistics, 34, 1265-1269. http://dx.doi.org/10.1214/aoms/1177703862

[38] AL-Hussaini, E.K. and Ahmad, K.E. (1981) On the Identifiability of Finite Mixtures of Distributions. IEEE Transactions on Information Theory, 27, 664-668. http://dx.doi.org/10.1109/TIT.1981.1056389

[39] Ahmad, K.E. (1988) Identifiability of Finite Mixtures Using a New Transform. Annals of the Institute of Statistical Mathematics, 40, 261-265. http://dx.doi.org/10.1007/BF00052342

[40] AL-Hussaini, E.K., Al-Dayian, G.R. and Adham, S.A. (2000) On Finite Mixture of Two-Component Gompertz Lifetime Model. Journal of Statistical Computation and Simulation, 67, 1-20. 